\title{
Kilowatt-range Picosecond Switching Based on Microplasma Devices
}

\author{
Mohammad Samizadeh Nikoo, Armin Jafari, Remco van Erp, and Elison Matioli, Member, IEEE
}

\begin{abstract}
Plasma formation in micro- and nano-scales enables an ultrahigh-dv/dt picosecond switching in an integrated circuit form factor. Such on-chip plasma devices could provide a transformative approach to achieving highpower levels at very high frequencies, far surpassing the best performance of conventional III-V electronics. In this work, we demonstrate the application of scaled-up nanoand microplasma switches as discrete devices in highperformance picosecond pulsed-power sources. A prototype circuit, utilizing the fabricated plasma devices operating as the switch, shows a high voltage-rising-rate beyond $10 \mathrm{kV} \mathrm{ns}^{-1}$ at $15 \mathrm{~kW}$ peak power. The pulsed-power source exhibits a sub-100 ps rise-time (without deembedding). The device has the capability of reaching exceptionally high current densities up to $325 \mathrm{~A} \mathrm{~mm}^{-1}$. The switches are compatible with planar semiconductor fabrication, enabling their integration with other electronic devices and microwave components, which opens pathways towards the future ultrahigh power density picosecond pulsed-power sources.
\end{abstract}

Index Terms-Ultrafast switch, picosecond, terahertz, nanoplasma, microplasma, pulsed-power, high-power.

\section{INTRODUCTION}

$\mathrm{F}$ ORMATION of plasma in micro- and nano-scales is gaining a growing attention both for fundamental researches and technological applications [1]. Such confined plasmas have been used for ultraviolet (UV) generation [2] and nanomaterial synthesis [3]. Recently, nano-scale plasma, socalled nanoplasma, has been shown to exhibit outstanding electronic functionality as a switch, providing an ultrafast operation thanks to the rapid electron transfer under high electric-fields [4]. The switch is normally-OFF and, by charging one of its terminals above the threshold voltage, undergoes an ultrafast switching in a few picoseconds by forming a plasma in micro/nano-scales (Figs. 1a and 1b). As a matter of fact, the real switching time of the device is still not known as the measured 5-ps was limited by the bandwidth of the measurement tools utilized [4].

The general operation of nanoplasma devices is similar to spark-gaps, however, the underlying physics is different, because the device dimension can be well below the mean-free path of electrons. This different physics together with a high electric-field, reaching $5 \mathrm{MV} \mathrm{cm}{ }^{-1}$, result in a rapid electron transfer and, therefore, an ultrafast picosecond switching time [4]. The device is compatible with planar fabrication techniques, which makes it capable of being monolithically integrated with other electronic devices. Compared to spark-

The authors are with the Power and Wide-band-gap Electronics Research Laboratory (POWERlab), École polytechnique fédérale de Lausanne (EPFL), CH-1015 Lausanne, Switzerland (mohammad.samizadeh@epfl.ch, elison.matioli@epfl.ch).
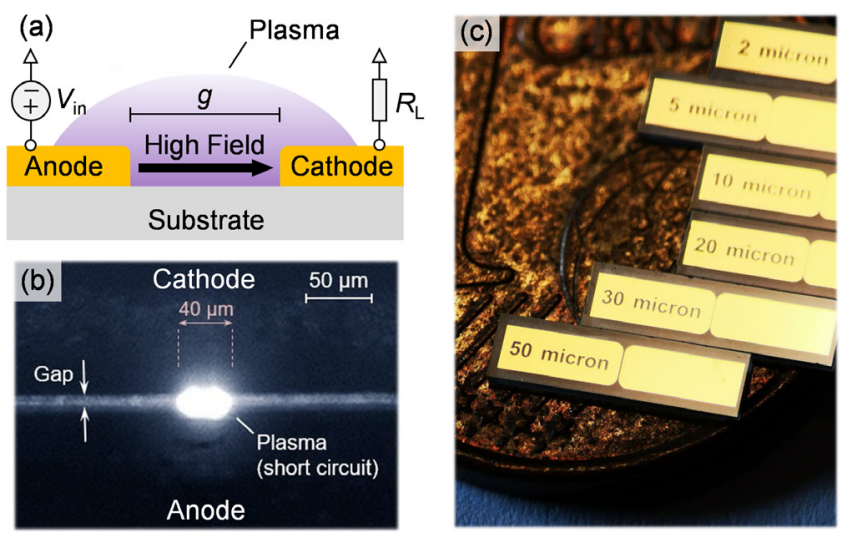

Fig. 1. (a) Schematic of the structure of a micro- or nanoplasma switch with gap size g. (b) Optical image of plasma formation in a micro-gap. The switch carries a 13-A peak current which considering the $40 \mu \mathrm{m}$ width of the plasma indicates a $325 \mathrm{~A} \mathrm{~mm}^{-1}$ current density. (c) Photograph of discrete switches with various gap distances.

gaps, nanoplasma switches can also be advantageous in terms of lifetime, thanks to their low ON-state voltage drop and potentially lower ionization level, as they can operate below mean-free path. A consistent and durable performance under harsh switching conditions has been demonstrated [4]. Using specific alloys or single crystalline metals can further improve the lifetime [5].

The fast switching dynamics of nanoplasma devices together with their high-frequency switching capability, over 10 $\mathrm{MHz}$ [4], and extremely high ON-state current that can go beyond $300 \mathrm{~A} \mathrm{~mm}^{-1}$, make them outstanding candidates for pulsed-power sources, where the switch plays a key role [6]-[9]. Conventional semiconductor devices, such as MOSFETs and HEMTs, although exhibit a versatile functionality with a practically infinite lifetime, suffer from a low on-state conductivity and a limited switching speed, below $1 \mathrm{kV}$ $\mathrm{ns}^{-1}$ [10], [11]. IGBTs are more widely used in pulsed power, however, their switching speed is very slow [12]. There are other electron devices than conventional transistors with fast switching capability, which are specifically developed for pulsed-power applications. For instance, the reverse recovery in drift-step-recovery diodes (DSRDs) interrupts the reverse current in a short time, thus acting as a fast switch [13].

Another example is the silicon avalanche sharpener (SAS) diode, which is a two-port device used for pulse sharpening [14]. Merensky et al. demonstrated 100-ps rise time $1.8-\mathrm{kV}$ pulse generator using a combination of DSRD and SAS [15]. Photoconductive semiconductor switches (PCSSs) also have a long history of use in sub-nanosecond pulsed-power switching [16]. The device takes advantage of opticallyinduced carrier generation driven by a high-power femtosecond laser, which makes the switching fast, but at the same time, 
costly and large. PCSS enables switching speeds in range of $\sim 10 \mathrm{kV} \mathrm{ns}^{-1}$. As an example, W. Shei et al. reported a 170-ps $1.3-\mathrm{kV}$ switching with $100-\mathrm{fs} 97.5-\mu \mathrm{J}$ triggering pulses using a GaAs PCSS [17]. In fact, all the aforementioned devices rely on special epitaxies that are not necessarily compatible with other semiconductors devices or microwave components, thus preventing their monolithic integration in more complex circuits. In addition, they are not typically commercially available.

Here we demonstrate the application of scaled-up microplasma switches for pulsed-power circuits. The devices are low-cost, easy-to-fabricate, and do not rely on multilayered epitaxies and special materials. In addition, although the switch can be used as discrete device (Fig. 1c), it offers the capability of integration, opening avenues for the future ultrahigh power density pulsed-power systems. We demonstrate a sub-100-ps fast switching speed reaching $14 \mathrm{kV} \mathrm{ns}^{-1}$ at $15 \mathrm{~kW}$ peak power.

\section{EXPERIMENTS AND RESULTS}

Fig. 2 shows the on-wafer switching characterization of nano- and microplasma devices with different gap sizes $(g)$ (Fig. 1a), ranging from $20 \mathrm{~nm}$ to $3000 \mathrm{~nm}$. The fabrication process of the switches started by a 100-nm-thick gold deposition (with 1-nm Ti adhesion layers on top and bottom) on a 2-inch Sapphire substrate. The nano-gaps were patterned by electron-beam lithography followed by an ion beam etching (IBE) step. Using photolithography and IBE we patterned the ground-signal-ground (GSG) radiofrequency pads. The devices were characterized by applying a low-speed signal with a $1-\mu \mathrm{s}$ rise time in the input, and the output was terminated by a $50-\Omega$ port of a 70-GHz oscilloscope (inset of Fig. 2). The electric connections between the device under test (DUT) and coaxial cables were done using radiofrequency probes. As shown in Fig. 2, the devices are initially in OFF state until the applied

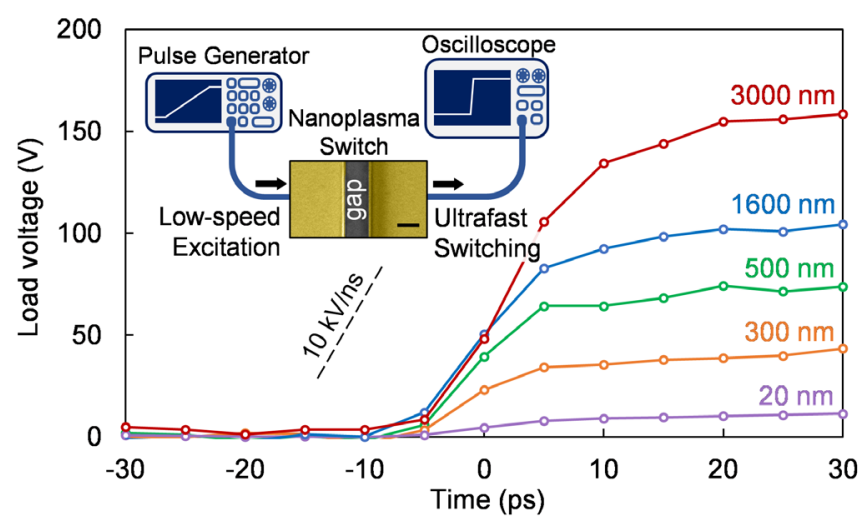

Fig. 2. On-wafer characterization of switching transient in nanoplasma devices with different gap sizes. The inset shows the experimental setup. The scale bar in the inset is $300 \mathrm{~nm}$.

voltage reaches the threshold voltage. At this point, the switch turns on in a few picoseconds due to plasma formation in the gap. In case of the 3000-nm gap, a 160-V 3.2-A switching corresponding to a $0.5 \mathrm{~kW}$ peak power was achieved. The power level can be further increased by increasing the gap distance, however, the voltage and current levels go well beyond the limitations of the employed radiofrequency probes.

To demonstrate the high-power switching capability of the plasma devices, we fabricated scaled-up switches with 1-mm wide channel and different gap distances, and employed the devices in an ultra-compact pulsed-power source. The scaledup devices were fabricated on a 1- $\mu \mathrm{m}$-thick tungsten deposited by sputtering on a 2-inch Sapphire substrate. The device layout was patterned by a single photolithography step followed by IBE. Then the wafer was diced into separate discrete devices. Based on such a simple fabrication process, the mass production of these switches can be very fast and low-cost.

Fig. 3a shows the experimental setup to evaluate the pulsedpower source utilizing the fabricated switches. A driver circuit
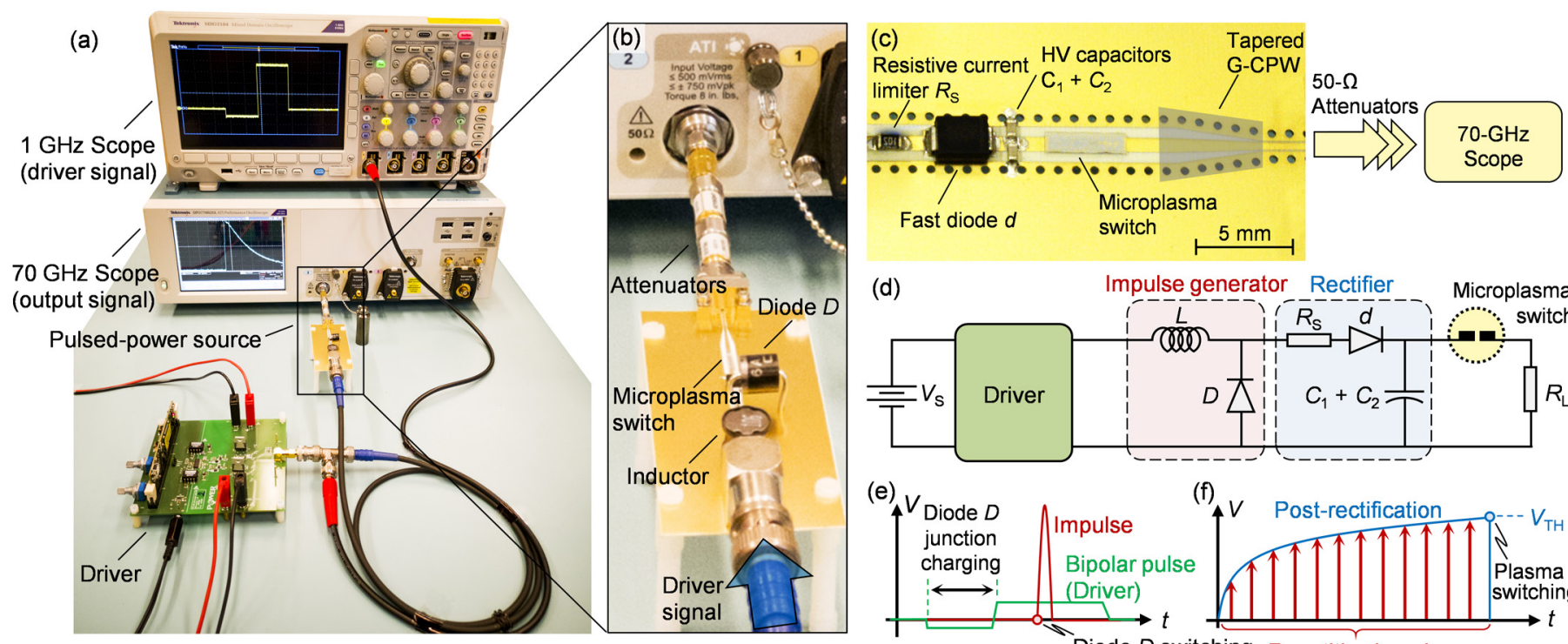

(d)
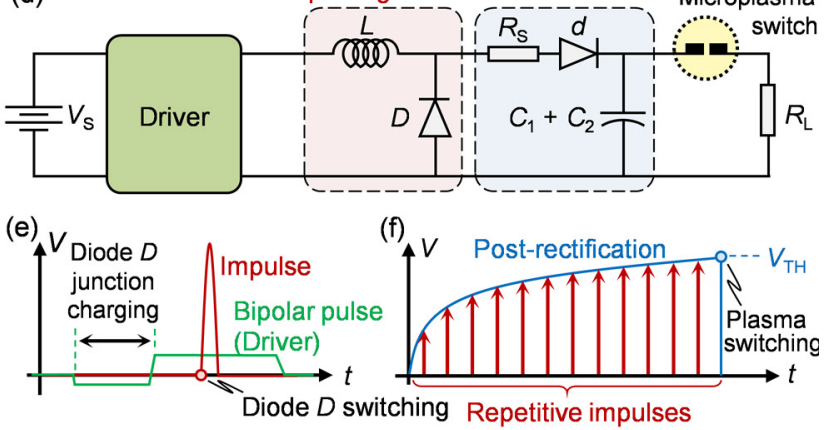

Fig. 3. (a) Photograph of the experimental setup to characterize (b) the ultra-compact pulsed-power source utilizing the fabricated plasma switches. Measurements were carried out with a 70-GHz real-time Tektronix DPO77002SX oscilloscope. (c) The top view of the circuit showing the current limiter resistor $R_{\mathrm{S}}$, fast diode $d$, high-voltage capacitors $C_{1}$ and $C_{2}$, microplasma switch, and a tapered G-CPW for output termination. (d) The schematic of the setup illustrating the driver circuit (the green PCB in part a), impulse generator, rectifier, microplasma switch, and the load $R_{\mathrm{L}}=50 \Omega$. Schematic of the (e) driver signal, impulse generated by the inductor-diode resonance, and (f) charging the HV capacitors by repetitive impulses until the micro-plasma switching.

(C) 2021 IEEE. Personal use of this material is permitted. Permission from IEEE must be obtained for all other uses, in any current or future media, including reprinting/republishing this material for advertising or promotional purposes, creating new collective works, for resale or redistribution to servers or lists, or reuse of any copyrighted component of this work in other works. 

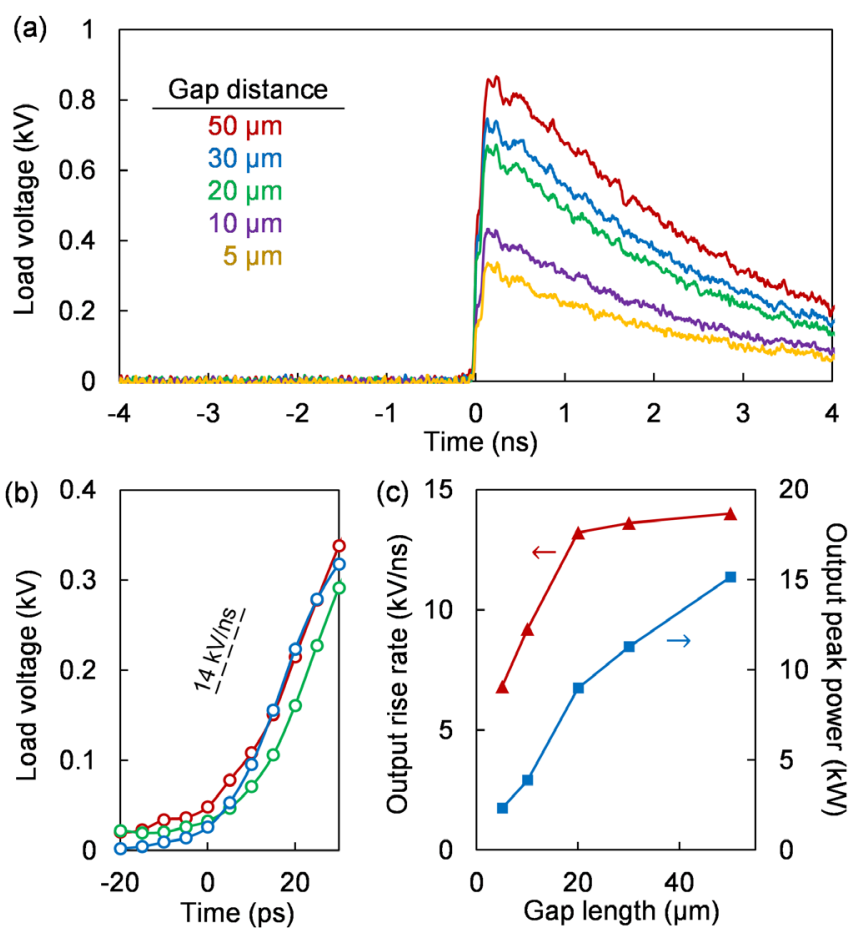

Fig. 4. (a) Voltage waveforms generated by the pulsed-power circuit. (b) Rising edge of the signals shown a high value of $\mathrm{d} v / \mathrm{d} t=14 \mathrm{kV} \mathrm{ns}^{-1}$.

(c) Output $d v / \mathrm{d} t$ and peak power for different gap lengths.

generates a bipolar waveform which is used as the excitation of the pulsed-power circuit (Figs. 3b and 3c). As shown in Fig. 3d, the initial stage of the pulsed-power circuit is an impulse generator which compresses the input bipolar pulse into a highamplitude short unipolar signal (Fig. 3e). The negative part of the bipolar signal biases the diode $D$ in the ON state. When the input signal turns positive, the diode $D$ does not immediately block the signal, because of the minority carriers stored in the junction [18]. When the charges are removed, the diode interrupts the current which initiates a resonance between inductor $L$ and the capacitance of the diode $D$, leading to the generation of impulse signals. The impulses charge the highvoltage capacitors through a rectifier stage (Fig. $3 f$ ).

The fabricated switch is then connected to the high-voltage capacitors. When the voltage across the capacitors reaches the threshold voltage $\left(V_{\mathrm{TH}}\right)$ of the plasma switch, it triggers the device and injects a high-speed pulse into the output port. The circuit is designed based on a grounded coplanar waveguide (GCPW) and the plasma switch is placed face-down, such that two ports of the device are in contact with the signal pads patterned in the printed circuit board (PCB). A $1.85-\mathrm{mm}$ connector rated for $67-\mathrm{GHz}$ bandwidth connects the GCPW to $67-\mathrm{GHz}$ coaxial attenuators with a total attenuation of $60-\mathrm{dB}$. The signal is finally terminated by the $50-\Omega$ port of a $70-\mathrm{GHz}$ oscilloscope.

Fig. 4a shows the output pulses generated by the pulsedpower circuit. We employed switches with different gap distances ranging from $5 \mu \mathrm{m}$ to $50 \mu \mathrm{m}$. The pulsed-power source generates very fast rising-edge output pulses at the $50-\Omega$ terminal, with a high $\mathrm{d} v / \mathrm{d} t$ reaching $14 \mathrm{kV} / \mathrm{ns}$, thanks to the high-speed operation of microplasma switches (Figs. $4 \mathrm{~b}$ and 4c). The output voltage reaches $870 \mathrm{~V}$ which corresponds to a peak current and power of $17.4 \mathrm{~A}$ and $15.1 \mathrm{~kW}$, respectively. For simplicity, we performed measurements on the ambient air, however, encapsulating the device under modified pressures [19] can be used to manipulate the threshold voltage, which would enable achieving higher powers in small gaps. In addition, a lower load resistance could be employed to absorb a much higher instantaneous power [20]; this is in particular interesting for microplasma switches as they can handle very high current levels.

It should be noted that, although the PCB is well designed, the parasitics in the path of output signal - from high-voltage capacitors to the coaxial connection - still affect the picosecond switching transient. For instance, measurements with ultra-low parasitic radiofrequency probes shows that the $\mathrm{d} v / \mathrm{d} t$ achieved by $3-\mu \mathrm{m}$-gap devices exceeds $11 \mathrm{kV} / \mathrm{ns}$ (Fig. 2), which is about two-times higher than the measured values in the compact pulsed-power source (Fig. 4c). This highlights the importance of the planar structure of on-chip plasma switches which enables a full integration to microwave components, such as transmission lines and antenna, to directly deliver the generated signal to the load.

\section{CONCLUSION}

This work showed that micro- and nanoplasma switches provide properties such as picosecond switching times and ultrahigh current densities that are highly desirable for pulsedpower applications. We characterized and employed 1-mmwide scaled-up switches with different gap distances for pulsedpower applications. A fast rising-edge of $14 \mathrm{kV} / \mathrm{ns}$ at peak power of $15 \mathrm{~kW}$ was obtained. The switching dynamic can be even further improved by an optimal circuit design. Very high performance and low-cost are advantages of micro- and nanoplasma switches compared to conventional semiconductor devices, with a trade-off of shorter lifetime than solid state devices. Ease of fabrication enables integration of nanoplasma switches with other electronic and microwave components which paves the way toward ultrahigh power-density all-onchip pulsed-power sources.

\section{REFERENCES}

[1] W. H.Chiang et al., "Microplasmas for advanced materials and devices," Advanced Materials, vol. 32, no. 18, 1905508, 2020. doi: 10.1002/adma.201905508.

[2] M. Raeiszadeh and F. Taghipour, "Microplasma UV lamp as a new source for UV-Induced Water treatment: protocols for characterization and kinetic study," Water Res., vol. 164, 114959, 2019. doi: 10.1016/j.watres.2019.114959.

[3] L. Lin, S. A. Starostin, S. Li, S. A. Khan, V. Hessel, "Synthesis of yttrium oxide nanoparticles via a facile microplasma-assisted process," Chem. Eng. Sci., vol. 178, 157- 166, 2018. doi: 10.1016/j.ces.2017.12.041.

[4] M. Samizadeh Nikoo, A. Jafari, N. Perera, M. Zhu, G. Santoruvo, and E. Matioli, "Nanoplasma-enabled picosecond switches for ultrafast electronics," Nature, vol. 579, no. 7800, pp. 534-539, Mar. 2020. doi: 10.1038/s41586-020-2118-y.

[5] S. Shingubara, Y. Nakasaki, and H. Kaneko, "Electromigration in a single crystalline submicron width aluminum interconnection," Appl. Phys. Lett., vol. 58, no. 1, pp. 42-44, Jan. 1991. doi: 10.1063/1.104431.

[6] S. N. Rukin, "Pulsed power technology based on semiconductor opening switches: A review," Rev. Sci. Instrum., vol. 91, no. 1, Jan. 2020, Art. no. 011501. doi: 10.1063/1.5128297.

[7] L. Li, Z. Zhao, Y. Liu, C. Li, J. Ren, and J. Li, "Repetitive gas-discharge closing switches for pulsed power applications," IEEE Trans. Plasma 
Sci., vol. 47, no. 9, pp. 4237-4249, Sep. 2019. doi: 10.1109/TPS.2019.2933660.

[8] X. Wang, S. K. Mazumder, and W. Shi, "A GaN-based insulated-gate photoconductive semiconductor switch for ultra-short high-power electric pulses," IEEE Electron. Device Lett., vol. 36, no. 5, pp. 493-495, May 2015. doi: 10.1109/LED.2015.2416188.

[9] A. Mojab and S. K. Mazumder, "Design and characterization of highcurrent optical Darlington transistor for pulsed-power applications," IEEE Trans. Electron Devices, vol. 64, no. 3, pp. 769-778, Mar. 2017. doi: 10.1109/TED.2016.2635632.

[10] E. Hoene, G. Deboy, C. R. Sullivan, and G. Hurley, "Outlook on developments in power devices and integration: Recent investigations and future requirements," IEEE Power Electron. Mag., vol. 5, no. 1, pp. 2836, Mar. 2018. doi: 10.1109/MPEL.2017.2782398.

[11] M. Samizadeh Nikoo, A. Jafari, and E. Matioli, "GaN transistors for miniaturized pulsed-power sources," IEEE Trans. Plasma Sci., vol. 47, no. 7, pp. 3241-3245, Jul. 2019. doi: 10.1109/TPS.2019.2917657.

[12] S. Mohsenzade, M. Zarghani, and S. Kaboli, "A high-voltage seriesstacked IGBT switch with active energy recovery feature for pulsed power applications," IEEE Trans. Ind. Electron., vol. 67, no. 5, pp. 36503661, May 2020, doi: 10.1109/TIE.2019.2921297.

[13] R. Sun et al. "10-kV 4H-SiC Drift Step Recovery Diodes (DSRDs) for Compact High-repetition Rate Nanosecond HV Pulse Generator," in Proc. Int. Symp. Power Semiconductor Devices ICs, Sept. 2020, pp. 62-65, doi: 10.1109/ISPSD46842.2020.9170132.
[14] I.V. Grekhov and A.F. Kardo-Sysoev, "Subnanosecond current drops in the delayed breakdown of silicon p-n junction," Sov. Tech. Phys. Lett., vol. 5 , no. 8, p. 395-396, 1979.

[15] L. M. Merensky, A. F. Kardo-Sysoev, A. N. Flerov, A. Pokryvailo, D. Shmilovitz, and A. S. Kesar, "A low-jitter 1.8-kV 100-ps rise-time 50$\mathrm{kHz}$ repetition-rate pulsed-power generator," IEEE Trans. Plasma Sci., vol. 37, no. 9, pp. 1855-1862, Sep. 2009. doi: 10.1109/TPS.2009.2025377.

[16] G. M. Loubriel, F. J. Zutavern, A. G. Baca, H. P. Hjalmarson, T. A. Plut, W. D. Helgeson, and M. W. O'Malley, M. H. Ruebush, and D. J. Brown, "Photoconductive semiconductor switches," IEEE Trans. Plasma Sci., vol. 25, no. 2, pp. 124-130, Apr. 1997. doi: 10.1109/27.602482.

[17] W. Shi, L. Yang, L. Hou, Z. Liu, N. Xu, and Z. Xing, "Positive and negative symmetric pulses with fast rising edge generated from a GaAs photoconductive semiconductor switch," Appl. Sci., vol. 9, p. 358, 2019. doi: 10.3390/app9020358.

[18] M. Samizadeh Nikoo and S. M. A. Hashemi, "High-power nanosecond pulse generator with high-voltage SRD and GDT Switch," IEEE Trans. Plasma Sci., vol. 43, no. 9, pp. 3268-3276, Sep. 2015. doi: 10.1109/TPS.2015.2411251.

[19] A. Hilton and D. S. Temple, "Wafer-level vacuum packaging of smart sensors," Sensors, vol. 16, no. 11, p. 1819, 2016. doi: 10.3390/s16111819.

[20] G. N. Appiah, S. R. Jang, J. S. Bae, C. G. Cho, S. H. Song, and H. J. Ryoo, "Compact design of high voltage switch for pulsed power applications," IEEE Trans. Dielectr. Elect. Insul., vol. 24, no. 4, pp. 2006-2013, Sep. 2017. doi: 10.1109/TDEI.2017.006272. 